\title{
Topical glyceryl trinitrate in the treatment of anal fissure
}

\author{
F. Novell, F. Novell-Costa and J. Novell \\ Service of General Surgery. Hospital Clínic. Barcelona. University of Barcelona, Spain
}

\begin{abstract}
Aim: the aim of this study was to assess the effectiveness of topical glyceryl trinitrate (GTN) in the treatment of anal fissure.

Patients: eighty consecutive patients were randomized to receive $0.2 \%$ GTN gel twice a day during a minimum of 6 weeks. Of these 80 patients 34 were males and 46 female, with ages between 20 and 78 years. All patients underwent a work-up that included gender, age, symptom duration, site, previous treatment attempts, pain score, associated diseases, and maximum anal resting pressure. These patients were followed at regular intervals of 4,8 , and 12 weeks to assess symptom outcome, rate of healing, adverse effects, and recurrence rate.

Results: pain scores were significantly reduced during the treatment period in $65 \%$ of cases. After 4 weeks, $55 \%$ of patients had healed, and 78\% after 9 weeks. In 18 patients (22\%), anal fissures did not heal even with 6 additional weeks of treatment, and 12 of them (15\%) underwent lateral sphincterotomy. Sixty one percent of patients had flushing and 15\% severe headaches.

Conclusion: the results of this study have demonstrated the significant benefit of topical GTN when administered to patients suffering from anal fissures.
\end{abstract}

Key words: Anal fissure. Gliceryl trinitrate. Surgical treatment.

Novell F, Novell-Costa F Novell J. Topical glyceryl trinitrate in the treatment of anal fissure. Rev Esp Enferm Dig 2004; 96: 255-258.

\section{INTRODUCTION}

Until recently, anal fissures were treated with diverse surgical procedures $(1,2)$, like anal dilation and lateral internal sphincterotomy, with the purpose of diminishing

Recibido: 09-06-03.

Aceptado: 22-09-03.

Correspondencia: F. Novell. Mallorca 309, 3º 08037 Barcelona. Telf.: 93 2074244. Fax: 932724831 the tension at the internal sphincter and to achieve its healing. However, both surgical treatments may cause varying degrees of anal incontinence in nearly $30 \%$ of cases. Recent studies have shown that nitric oxide donor derivatives like glyceryl trinitrate (GTN) may reduce the pressure of the sphincter and achieve the healing of the fissure.

The objective of this study was to evaluate results obtained with GTN in the treatment of anal fissure (3-5).

\section{MATERIAL Y METHOD}

A total of 80 consecutive patients with anal fissure were treated with $0.2 \%$ GTN twice a day for a minimum of 6 weeks. Thirty-four patients were males and 46 were females. Their age ranged from 20 to 78 years, and their clinical characteristics are listed in table I. All patients were monitored on an outpatient basis.

According to outcome, fissures were classified as: a) acute; b) progressive -more than 1 month of evolution, thick borders, and sentry hemorrhoid; and c) chronic anal fissures, evolving through several months with marginal induration, sphincter muscle fiber exposure, great sentry hemorrhoid, and failure of previous treatments.

The pain score was checked using a visual analog scale (VAS) for absent, mild, moderate, severe or maximum pain.

All patients were monitored at regular intervals of 4,8 and 12 weeks in order to control their symptoms, fissure healing, adverse effects, and potential relapse.

\section{RESULTS}

The results of GTN administration are shown in table II.

There were 69 posterior and 11 anterior fissures. Pain severity decreased to mild when evaluated with the VAS at 3 days after treatment onset in 42 patients. 
Table I. Clinical characteristics

\begin{tabular}{l} 
Number of cases in relation to the age \\
6 cases between 20 and 30 years \\
14 cases between 31 and 40 years \\
26 cases between 41 and 50 years \\
34 cases in old 51 years. \\
Type of fissures \\
10 cases acutes \\
49 cases evolved \\
21 cases anal chronic fissures \\
Localization \\
11 cases (13,7 \%) in anterior site \\
69 cases ( $86,3 \%$ ) in posterior site \\
Symptoms in the exploration \\
Haemorrhage in $75.6 \%$ of cases \\
Pain in $100 \%$ of cases \\
Rectal tenesmus in $24,3 \%$ of cases \\
Itching in $22 \%$ of cases \\
Sphincter spasm in $92.6 \%$ of cases. \\
Associate pathology \\
Hemorrhoids, 18 cases \\
Hemorrhoids 6 cases, previous surgery of hemorrhoids \\
1 case, previous intervention of anal fistulous \\
6 cases, previous internal sphincterotomy \\
1 case of anal fissure. Previous manual anal dilation. \\
\hline
\end{tabular}

Table II. Results topic treatment with GTN

Tolerance to GTN
49 cases, light headache
12 cases, severe headache
3 cases, orthostatic hypotension
Results according as fissure types
Acutes: 10 , all cases healed
Evolves: $49 ; 45$ healed, and 3 incomplete healed
Chronic: $21 ; 7$ healed, 12 failure treatment, 2 healed incomplete
Complete healing, $62(78 \%)$ cases
Incomplete healing, $6(73 \%)$ cases
Wanted surgery, $12(15 \%)$ cases

At week 4, 44 patients had achieved a complete healing, and 62 achieved this on the 9th week. GTN treatment failed in 18 patients, 15 with chronic fissures and 3 with progressive fissures. Twelve of these 18 patients subsequently underwent lateral sphincterotomy. Six patients, 5 with chronic fissures and 1 with progressive fissures, who had incomplete healing rejected another treatment because of minor nuisances.

Forty-eight patients had flushing and mild headache after GTN administration. No patient required treatment discontinuation because of adverse effects, despite the fact that 12 subjects had severe headache. Three patients had orthostatic hypotension fol-lowing the first application of GTN.

\section{DISCUSSION}

Until recently the treatment of anal fissure was surgical $(6,7)$, but the moderate prevalence of complications such as anal incontinence or abscess has promoted other treatments $(8,9)$, like topical $0.2 \%$ GTN $(10-13)$.

GTN is an nitric oxide donor that increases local blood flow, contributes to the relaxation of the internal sphincter, and decreases resting pressure at the anal canal. Its topical application acts like a "chemical sphincterotomy" and is able to cure the fissure.

The evaluation of blood flow at the anal canal has been shown to be increased very significantly in $65 \%$ of patients treated with GTN (14), and the pressure at this level decreased by $40 \%$ of previous values within 20 minutes following GTN $(15,16)$. This decrease maintained at least for 90 minutes.

In responsive subjects, anal pain usually begins to decrease within 3-5 days of treatment, and healing is achieved between weeks 4 and 9. GTN treatment failure is due to poor GTN gel administration, to the presence of marked fibrosis at the fissure, or to sphincter spasm.

Seventy-six percent of our patients showed flushing and headache (17-19).

When related to age, incidence started from 50 years on. Regarding our patients, $12.5 \%$ had acute anal fissures, $61.25 \%$ had progressive fissures, and $26.25 \%$ had chronic anal fissures. In $40 \%$ of treated cases there was some associated anal condition.

In conclusion, these results demonstrate a significant benefit from the topical administration of $0.2 \%$ GTN to anal fissures.

\section{REFERENCES}

1. Richard CS, Gregoire R, Plewes EA, Silverman R, Burul C, Buie D, et al. Internal sphincterotomy is superior to topical nitroglycerin in the treatment of chronic anal fissure: results of a randomized controlled trial by the Canadian Colorectal Trials Group. Dis Colon Rectum 2000; 43: 1048-57.

2. Nelson RL. Meta-analysis of operative techniques for fissure-in-ano. Dis Colon Rectum 1999; 42: 1424-8.

3. Watson SJ, Kamm MA, Nichols RJ, Phillips RK. Topical glyceryl trinitrate in the treatment of chronic anal fissure. Br J Surg 1996; 83: 771-5.

4. Lund JN, Armitage NC, Scholefield JH. Use of gliceryl trinitrate ointment in the treatment of anal fissure. Br J Surg 1996; 83: 776-7.

5. Palazzo FF, Kapur S, Steward M, Cullen PT. Glyceryl trinitrate of chronic fissure in ano: one year's experience with $0.5 \%$ GTN paste. J R Coll Surg Edinb 2000; 45: 168-70.

6. Christei A, Guest JF. Modelling the economic impact of managing a chronic anal fissure with a proprietary furmulation of nitroglycerin (Rectogesic) compared to lateral internal sphincterotomy in the United Kingdon. Int J Colorectal Dis 2002; 17: 259-67.

7. Libertiny G, Knight JS, Farok R. Randomized trial of topical $0.2 \%$ glyceryl tinitrate and lateral internal sphincterotomy for the treatment of patients with chronic anal fissure long-term follow-up. Eur J Surg 2002;1 68: 418-21.

8. Brisinda D, Maria G, Fenici R, Civello IM, Brisinda G. Safety of botulinum neurotoxin treatment in patients with chronic anal fissure. Dis Colon Rectum 2003; 46: 419-20.

9. Madalinski MH, Slawek J. The higher doses of botulinum toxin and 
the potential effects of its action after nitric oxide donors application for the treatment of chronic anal fissure. Surgery 2003; 133: 455-6.

10. Scholefield JF, Bock JU, Marala B, Richter HJ, Athanasiadis S, Prols $\mathrm{M}$, et al. A dose finding study with $0.1 \%, 0.2 \%$, and $0.4 \%$ glyceryl trinitrate ointment in patients with chronic anal fissures. Gut 2003; 52: 264-9.

11. Svendsen CB, Matzen P. Treatment of chronic anal fissure with topically applied nitroglycerin ointment. A systematic reviwe of evidence- based results. Ugeskr Laeger 2002; 164: 3845-9.

12. Graziano A, Svider L, Lencinas S, Masacangiol G, Gualdrini U, Bisisio $\mathrm{O}$. Long -term results of topical nitroglycerin in the treatment of chronic anal fissures are disappointing. Tech Coloproctol 2001; 5: 143-7.

13. Kua KB, Kocher HM, Kelkar A, Patel AG. Effect of topical glyceryl tinitrate on anodermal blood flow in patients with chronic anal fissures. ANZ J Surg 2001; 71: 548-50.
14. Jonas M, Amin S, Wright JW, Neal KR, Scholefield JH. Topical 0.2 percent glyceril trinitrate ointment has a short-lived effect on resting anal pressure. Dis Colon Rectum 2001; 44: 1640-3.

15. Pitt J, Williams S, Dawson PM. Reasons failure of glyceryl trinitrate treatment of chronic fissure-in-ano: a multivariate analysis. Dis Colon rectum 2001; 46: 846-7.

16. Moorthy K, Chavez R. Audit of topical glyceryl trinitrate for treatment of fissure-in-ano. Ann R Coll Surg Engl 2001; 83: 220-1.

17. Ward DI, Miller BJ, Schache DJ, Cohen JR, Theile DE. Cut or paste? The use of glyceryl trinitrate paste in the treatment of acute and chronic anal fissure. Aust N Z J Surg 2000; 70: 19-21.

18. Hasegawa H, Radley S, Morton DG, Dorricott NJ, Campbell DJ, Keighley MR. Audit of topical glyceryl trinitrate for treatment of fissure-in-ano. Ann R Coll Surg Engl 2000; 82: 27-30.

19. Mc Leod RS, Evans J. Symptomatic care and nitroglycerin in the management of anal fissure. J Gastrointest Surg 2002; 6: 278-80.

\title{
Utilidad del gliceril trinitrato en el tratamiento tópico de la fisura anal
}

\author{
F. Novell, F. Novell-Costa y J. Novell \\ Servicio de Cirugía General. Hospital Clínic. Barcelona. Universidad de Barcelona
}

\section{RESUMEN}

Objetivo: el objetivo de este estudio era la valoración de la aplicación tópica de gliceril trinitrato en el tratamiento de las fisuras anales.

Pacientes: un total de 80 consecutivos pacientes eran randomizados recibiendo gliceril trinitrato al $0,2 \%$ gel dos veces al día, durante un mínimo de 6 semanas. De los 80 pacientes, 34 eran varones y 46 mujeres, con edad comprendida entre 20 y 78 años. En los pacientes se controlaba sexo, edad, duración de síntomas, duración de tratamiento, localización, patología previa asociada, y valoración del dolor. Seguimiento a 4, 8 y 12 semanas, para controlar la sintomatología, el grado de curación, los efectos adversos y la relación de recidiva.

Resultados: la disminución del dolor fue sionificativa en el $65 \%$ de los casos y se consiguió la curación de la fisura a las 4 semanas en el 55\% de los pacientes, y el 78\% a las 9 semanas de tratamiento. El tratamiento falló en $18(22 \%)$ casos, requiriendo intervención quirúrgica en 12 (15\%) de ellos. Un 61\% de los pacientes refirieron cefalea leve y rubicundez facial tras la aplicación de GTN, y en 12 (15\%) pacientes la cefalea era intensa.

Conclusión: estos resultados han demostrado el significativo beneficio de la aplicación tópica de gliceril trinitrato al 0,2\% en las fisuras anales.

Palabras clave: Fisura anal. Gliceril trinitrato. Tratamiento quirúrgico.

\section{INTRODUCCIÓN}

Hasta hace poco tiempo la fisura anal (FA) se ha tratado con diversas técnicas quirúrgicas $(1,2)$, como dilata- ción anal y esfinterotomía lateral interna, con el fin de disminuir la tensión en el músculo esfínter interno y con ello lograr su curación. Sin embargo, ambos tratamientos quirúrgicos pueden causar un grado de incontinencia esfinteriana por encima del $30 \%$ de los casos. Estudios recientes han mostrado que los derivados del óxido nítrico, como el gliceril trinitrato (GTN) pueden reducir la presión del esfínter y lograr la curación de la fisura (3-5).

El objetivo de este estudio es la valoración de los resultados con GTN 0,2\% en el tratamiento de la fisura anal.

\section{MATERIAL Y MÉTODO}

Un total de 80 pacientes consecutivos con fisura anal eran randomizados recibiendo gliceril trinitrato al $0,2 \%$ gel dos veces al día, durante un mínimo de 6 semanas. De los 80 pacientes, 34 eran varones y 46 mujeres, con edad comprendida entre 20 y 78 años, y cuyas características clínicas se exponen en la tabla I. Se incluyeron todos los pacientes asistidos en consulta.

Según su evolución, las fisuras eran clasificadas como: a) recientes; b) evolucionadas, a partir de un mes de evolución, bordes espesos y hemorroide centinela; y c) crónicas, de varios meses de antigüedad, con induración de los bordes de la fisura, exposición fibras del esfínter, gran hemorroide centinela, y fracaso tratamientos previos.

El efecto del tratamiento sobre el dolor era valorado apli- 
cando la escala analógica visual (EAV), con ausencia de dolor, leve, moderado y severo, y máximo dolor imaginable.

Todos los pacientes fueron revisados a intervalos regulares de 4, 8, y 12 semanas para controlar la sintomatología, el grado de curación, los efectos adversos y las posibles recidivas.

\section{Tabla I. Características clínicas}

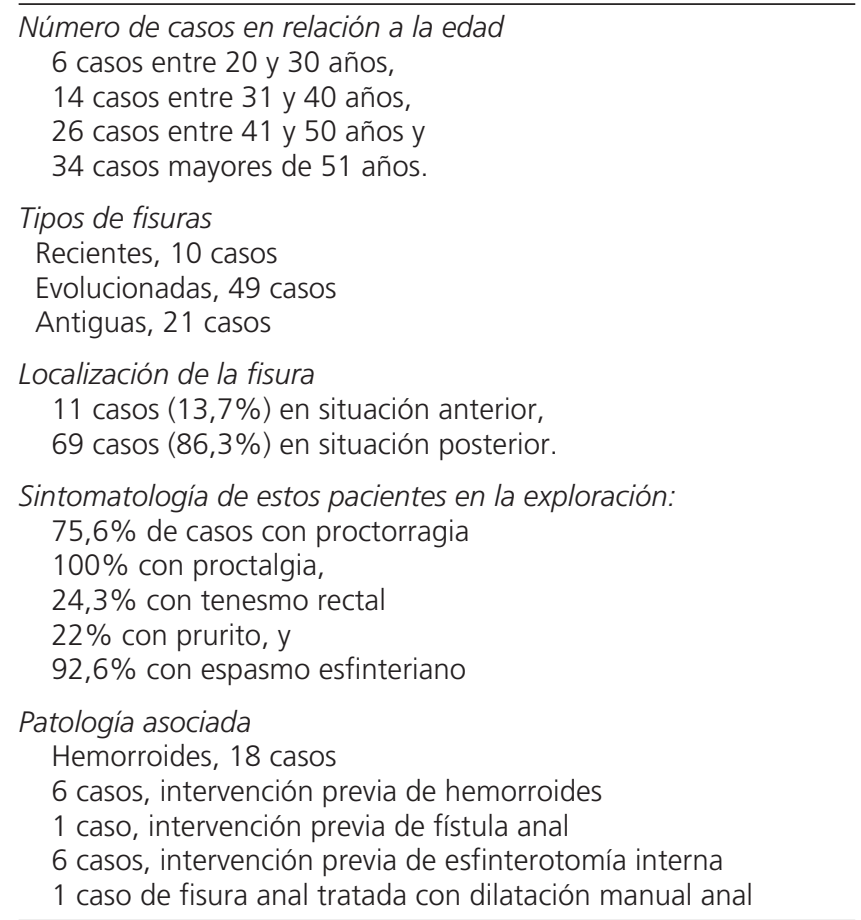

\section{RESULTADOS}

Los resultados de la aplicación de GTN se exponen en la tabla II.

El dolor disminuyó de intensidad hasta leve según la EAV a partir del tercer día en $42(65 \%)$ de los pacientes.

Se consiguió la curación de la fisura en $44(55 \%)$ de los pacientes a las 4 semanas, y en $62(78 \%)$ casos a las 9 semanas. El tratamiento con GTN falló en 18 (22\%) pacientes, 15 fisuras crónicas y 3 evolucionadas, requiriendo intervención quirúrgica en $12(15 \%)$ de ellos. En 6 pacientes, 5 fisuras crónicas y 1 evolucionada, aunque la curación fue incompleta, los pacientes rechazaron otro tratamiento por las pocas molestias que les ocasionaban.

Cuarenta y ocho $(61 \%)$ pacientes presentaron rubicundez facial y cefalea leve tras la aplicación de GTN, que no requirieron medicación ni la suspensión del tratamiento, y en $12(15 \%)$ casos la cefalea fue intensa. Tres $(3,7 \%)$ pacientes presentaron hipotensión ortostática tras la primera aplicación de GTN.

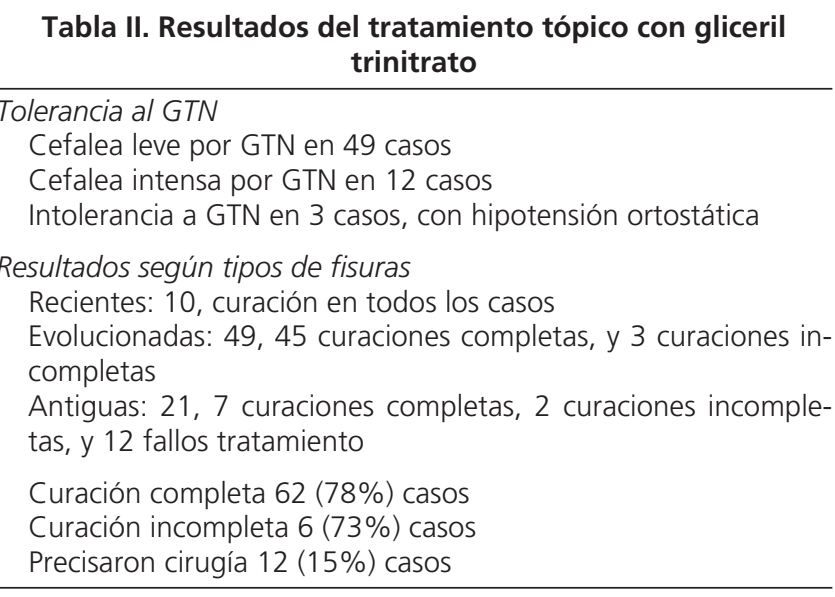

\section{DISCUSIÓN}

Hasta hace poco tiempo el tratamiento de la fisura anal era quirúrgico $(6,7)$, pero por la prevalencia de complicaciones como incontinencia anal, abscesos, fístula y hematomas, se han aplicado otros tratamientos $(8,9)$, como la aplicación tópica de gliceril trinitatro al 0,2\% (10-13).

El GTN es un neurotransmisor que origina un aumento del flujo sanguíneo local, contribuye a la relajación del músculo esfínter interno, y disminuye la presión de reposo en el canal anal. Su aplicación tópica actúa como una "esfinterotomía química" capaz de curar la fisura.

La valoración del aumento del flujo sanguíneo en el canal anal se ha mostrado muy significativo en el $65 \%$ de los pacientes tratados con GTN (14) y la disminución de la presión del conducto anal (MRP) hasta en un $40 \%$ de los valores previos a los 20 minutos de la aplicación local de GTN, manteniéndose dicha presión anal en reposo durante 90 minutos $(15,16)$.

El dolor anal en los casos favorables suele remitir a los 3-5 días, y la curación suele lograrse entre 4 y 9 semanas. Los casos en que el tratamiento con GTN ha fracasado han sido debidos a la mala aplicación del gel de GTN, a la presencia de fibrosis acentuada de la fisura, y a la presencia de espasmo esfinteriano.

En el $76 \%$ de los casos hemos observado rubicundez facial y cefalea, de intensidad variable (17-19).

En relación a la edad, la mayor incidencia es a partir de los 50 años. En nuestros casos, el 12,5\% correspondían a fisuras anales recientes, el 61,25\% a fisuras evolucionadas, y el 26,25\% a fisuras crónicas. En el $40 \%$ de los casos tratados había patología asociada del canal anal.

En conclusión, estos resultados han demostrado el significativo beneficio de la aplicación tópica de gliceril trinitrato gel al $0,2 \%$ en las fisuras anales. 